\title{
Dielectric Constant Measurements of Pure and Nickel Sulphate Doped KDP Crystals grown by Gel Medium
}

\author{
M.P.Rameela ${ }^{1}$, Vinu Bharathi ${ }^{3}$ T.H.Freeda ${ }^{2}$ and T. Asaithambi ${ }^{3}$ \\ ${ }^{I}$ Department of Physics,Sarah Thucker College For Women, Thirunelveli - 627007. \\ ${ }^{2}$ Department of Physics,S.T.Hindu College Nagercoil,Kanyakumai Dist \\ ${ }^{3}$ P.G and Research Department of Physics, Alagappa Govt.Arts College Karaikudi - 630003
}

\begin{abstract}
Crystal growth is an interdisciplinary subject covering physics, chemistry, material science, chemical engineering, metallurgy, crystallography, mineralogy, etc. In past few decades, there has been a growing interest on crystal growth processes, particularly in view of the increasing demand of materials for technological applications. Optically good quality pure and metal doped KDP crystals have been grown by microbial free gel growth method at room temperature and their characterization have been studied. Gel method is a very simple method and can be utilized to synthesize crystals which are having low solubility. Potassium dihydrogen orthophosphate KH2PO4 (KDP) continues to be an interesting material both academically and industrially. KDP is a representative of hydrogen bonded materials which possess very good electro - optic and nonlinear optical properties in addition to interesting electrical properties. Due to this interesting properties, we made an attempt to grow pure and nickel sulphate doped kdp crystals in various concentrations (0.002, 0.004, 0.006, 0.008 and 0.010) using gel method. The grown crystals were collected after 20 days. We get crystals with good quality and shaped. The dielectric constant values were measured at different frequencies of pure and nickel sulphate added crystal with a temperature range of $40^{\circ} \mathrm{C}$ to $120^{\circ} \mathrm{C}$ using simple two probe setup with $Q$ band digital LCR meter present in our lab. The electrical conductivity increases with increase of temperature. The dielectric constants value of metal doped KDP crystals were slightly decreased compared to pure KDP crystals.
\end{abstract}

\section{Introduction}

KDP is a representative of hydrogen bonded materials which possess very good electro - optic and nonlinear optical properties in addition to interesting electrical properties. The demand for high quality large single crystals of KDP increase due to the application as frequency conversion crystal in inertial confinement fusion [1-2]. The piezo electric property of KDP crystal makes it useful for the construction of crystal filters and frequency stabilizers in electronic circuit's. The excellent properties of KDP include transparency in a wide region of optical spectrum, resistance to damage by laser radiation and relatively high non- linear efficiency, in combination with reproducible growth to large size. Therefore, it is commonly used in several applications such as laser fusion, electro-optical modulation and frequency conversion. Many studies on the growth and properties of KDP crystals in the presence of impurities have been reported [3 - 4]. Potassium dihydrogen phosphate (KDP) crystal draws persistent attention of scientists due to its excellent quality and possibility of growing large- size crystals [5 - 6]. Microscopically, crystal growth includes crystal morphology, crystal defects, and growth rate, which are all related to the constituent growth units and their chemical bonding process [7 - 8]. KDP, ADP and DKDP are the only nonlinear crystal currently used for these applications due to their exclusive properties. The grown crystals were characterized using dielectric constant, electrical properties, optical transmittance, for pure and $\mathrm{NiSO}_{4}$ doped KDP crystals.

\section{Materials and Methods}

Pure and $\mathrm{NiSO}_{4}$ doped KDP single crystals are grown in sodium meta silicate gel medium using analar grade KDP and NiSO4 with in concentrations of 0.002, 0.004, 0.006, 0.008 and 0.010 of dopant and sodium meta silicate $\left(1.08 \mathrm{~g} / \mathrm{cm}^{3}\right)$. During the process $\mathrm{p}^{\mathrm{H}}$ was maintained at $5-6$ at room temperature figure (1). Ethyl alcohol of equal volume is added over the set gel without damaging the cell surface. When the alcohol diffuses into the set gel, it reduces the solubility. This induces nucleation and the nuclei are grown into the single crystals. The crystal growth was carried out at room temperature. The growth period was about 20 days for pure and $\mathrm{NiSO}_{4}$ doped $\mathrm{KDP}$ crystals. 

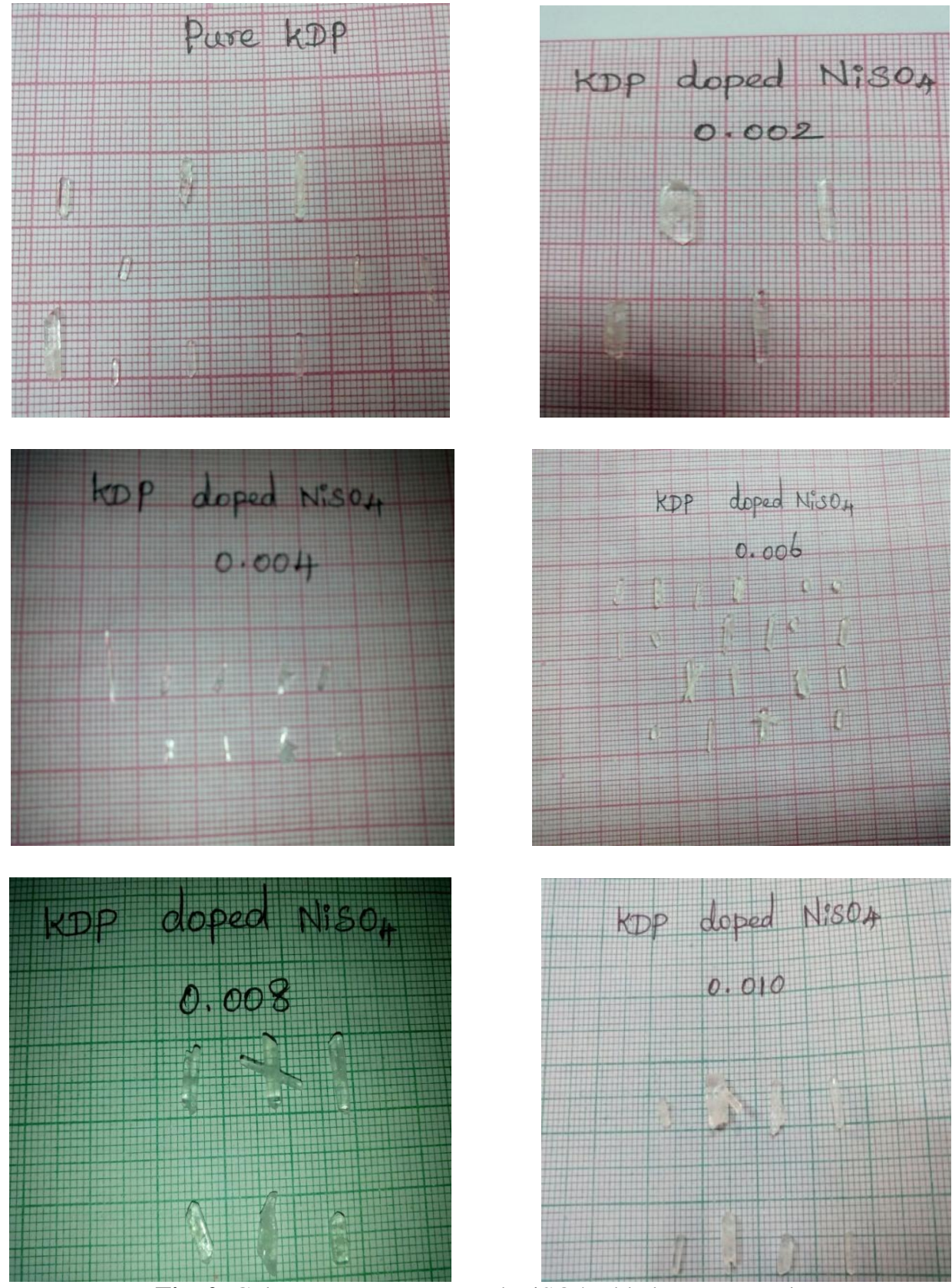

Fig. 2. Gel grown Pure KDP and NiSO4 added KDP crystals

Doping: Doping means adding impurity to the known pure crystal .To prepare a doped crystal a required amount of dopant solute is also mixed along with the pure solute.

Table 1: Doping concentrations of impurities

\begin{tabular}{|c|c|}
\hline Doping ratio & Mass of NiSO $_{4}$ Added in gm \\
\hline $1: 0.002$ & 0.06571 \\
\hline $1: 0.004$ & 0.13143 \\
\hline $1: 0.006$ & 0.19714 \\
\hline $1: 0.008$ & 0.26286 \\
\hline $1: 0.010$ & 0.32857 \\
\hline
\end{tabular}

An impurity can suppress, enhance or stop the growth of crystal completely. It usually acts on certain crystallographic faces. The effects depend on the impurity concentration, super saturation, temperature and $\mathrm{pH}$ of the solution. 
Dielectric Constant Measurements of Pure and Nickel Sulphate Doped KDP Crystals grown by..

\section{Dielectric constant studies}

\section{Results and Discussions}

Dielectric properties are correlated with electro- optic properties of the crystals particularly when they are non- conducting materials. Due to the incorporation of metal ions polarization increases and the electrical conductivity increases. (fig.5,6) The magnitude of dielectric constant depends on the degree of polarization, charge displacement in the crystal. The dielectric constant of materials is due to the contribution of electronic, ionic, dipolar and space charge polarizations which depends on the frequencies [5]. At low frequencies, all these , polarization are active lower frequencies and high temperatures [6], in KDP crystals, many reports are available about its dielectric behavior and in our present work the measured dielectric constant values are in good agreement with the reported results [7-8]. The temperature dependence of dielectric constant at frequency $100 \mathrm{~Hz}$ to $1 \mathrm{KHz}$ is shown in figs 3 and 4 . Even though KDP has many reports on dielectric loss, the study clearly ensures the crystalline perfection of crystals in our present case; it is observed that the dielectric loss decreases with increasing frequency and low dielectric losses were observed for the crystals grown by gel method compared to the solution growth. The lower value of dielectric constant is a suitable parameter for the enhancement of SHG signals. The measurement of dielectric constant and loss as a function of frequency at different temperatures give an idea about the electrical processes that are taking place in materials and these parameters were measured on the polished (010) face of the pure and metal doped KDP crystals. Frequency dependences of dielectric constant of these crystals at room temperature are observed from the figures and it is observed that dielectric constant of KDP and $\mathrm{NiSO}_{4}$ doped KDP crystals are high at low frequencies and they decreases with increase in frequency. The very high value of $\mathrm{\epsilon r}$ at low frequencies may be due to the presence of all four polarizations namely space charge, orientation, electronic and ionic and its low values at higher frequencies may be due to the loss of significance of these polarizations gradually. The nature of decrease of $\epsilon \mathrm{r}$ frequency suggests that pure and $\mathrm{NiSO}_{4}$ doped KDP crystals contain dipoles of continuously varying relaxation times.

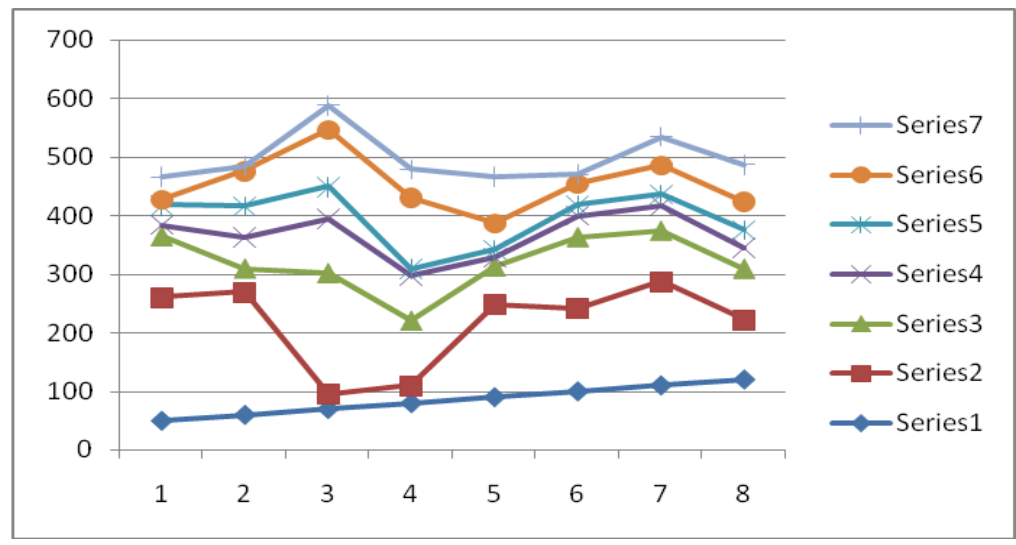

Fig.3. Variation of dielectric constant $\left(\varepsilon_{\mathrm{r}}\right)$ with temperature at frequency of $100 \mathrm{~Hz}$ for pure and $\mathrm{NiSO}_{4}$ doped KDP crystals

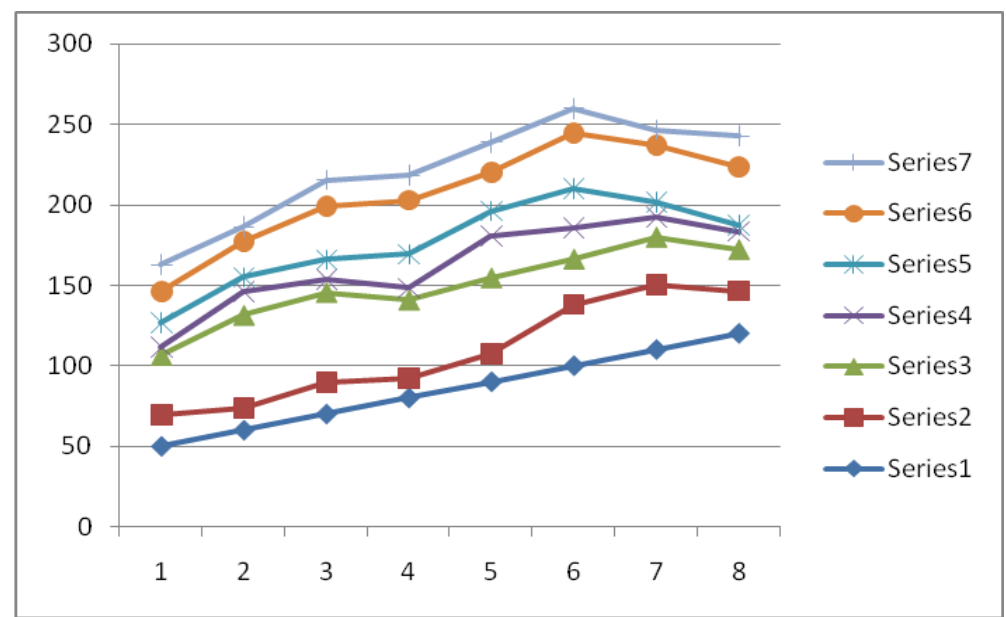

Fig.4. Variation of dielectric constant $\left(\varepsilon_{\mathrm{r}}\right)$ with temperature at frequency of $1 \mathrm{KHz}$ for pure and $\mathrm{NiSO}_{4}$ doped KDP crystals 


\section{Conclusions}

Temperature has a striking effect on dielectric properties. The trend of dielectric constant and electrical conductivity as a function of frequency is the same for all temperatures for both pure and doped crystals. It is observed that the dielectric constant and dielectric loss slightly increase with the temperature. This phenomenon can be explained as follows: The electronic polarizations. Increase of with the increase of temperature. The increase of temperature of the crystal influences the ionic and electronic polarizations. The frequency dependence of dielectric constant and dielectric loss at temperatures beyond $100^{\circ} \mathrm{C}$ can be again attributed to space charge polarization.

\section{References}

[1]. X.Sun, X, Xu, Z.Gao, Y.Fu, S.Wahg, H.Zong, Y.Li, J.Cryst.Growth., 217(2000) 404

[2]. $\quad$ N.Zaitseva, L.Carman, I.Smolsky, J.Cryst.Growth., 241 (2002) 363.

[3]. Bo Wang. Chang - ShuiFang,Sheng - Iai Wang, Xun Sun, Quing-tianGu,Yi-ping Li, Xin-guangXu, JianqinZhang,BingLiu, KDP doped with potassium carbonate as additives, .J.Cryst.Growth ,310, (2008) 5341-5346.

[4]. N.Pattanaboonmee, P.Ramasamy, P.Manyum, Procedings of Engineering., 32 (2012) 1019-1025.

[5]. Dongli Xu, Dongfeng Xue. J.Cryst. Growth .,310 (2008) 1385

[6]. Dogli Xu, Dongfeng Xue, J.Alloys Compd., 449 (2008) 353

[7]. Dongli Xu, Dongfeng Xue. J.Cryst. Growth .,310 (2008) 1385

[8]. S.Balamurugan, P.Ramasamy, Spectrochimica Acta., Part A 71 (2009) 1979-1983 